\title{
TRATAMENTO DA GIARDIASE COM TINIDAZOL *
}

\author{
Miroslau Constante Baranski**, Milton Carneiro Filho ***, Luiz Alberto Cagliari \\ Santos $* * * *$ e Cláudio José Trezub *****
}

\begin{abstract}
Estudou-se a eficácia terapêtica do tinidazol, um novo derivado nitroimidazólico, em trinta pacientes com giardíase. A posologia diária foi de $300 \mathrm{mg} \mathrm{em}$ 20 pacientes com idade superior a 10 anos, e de $150 \mathrm{mg} \mathrm{em} 10$ crianças com idade inferior a 10 anos. O tratamento teve a duração de cinco dias.

o diagnóstico e os controles de cura parasitológicos foram feitos pelos métodos de Hoffman, Pons \& Janer $e$ de Faust e cols. Foram realizados três controles, com intervalos de sete dias, iniciando-se na semana subsequente ao término do tratamento.

Obtiveram cura parasitológica em $96,6 \%$ dos casos. Ressaltam a excelente tolerância da droga, mesmo em pacientes com grave doença fundamental ou em crianças, não se registrando efeitos colaterais.

Concluem ser o tinidazol excelente droga giaraicida.
\end{abstract}

\section{INTRODUÇÃO}

O armamentário terapêutico contra a infecção ou a doença produzida pelo protozoário flagelado Giardia lamblia foi enriquecido, nos últimos anos, pela introdução dos muito eficazes derivađos nitroimidazólicos.

Dentre eles, o metronidazol e a nitrimidazina foram objeto de outras investigações nossas $(15,5)$ e são os que mereceram até então, a preferência da maioria dos médicos.

Não há mais aúvida de que a Giardia lamblia é dotada de evidente ação patogênica, após estudos realizados com biópsias intestinais e microscopia eletrônica (13).

A incidência da giardíase no Estado do Paraná varia entre 8 e $24 \%$, de conformidade com o grupo populacional estudado a dos métodos empregados nas investigações parasitológicas $(1,4,6,8,11$ e 12). Em 6900 exames parasitológicos de fezes efetuados no Hospital das Clínicas da Universidade Federal ao Paraná, um dos autores (M. C Fo, 6). assinalou $8 \%$ de positividade para cistos de Giardia lamblia, empre. ganáo os métodos de Faust e cols. e de Hoffman, Pons \& Janer.

$\mathrm{Em}$ prosseguimento a anteriores investigações clínico-terapêuticas no campo das enteroparasitoses, e sobretudo tendo em conta a excelente atividade tricomonícida, por via sistêmica, de um novo derivado nitrcimidazólico - o Tinidazol - resolvemos empregá-lo em pacientes infectados pela Giardia lamblia.

De acordo com estudos realizados por Howes Jr. \& Cols. (7), Miller \& Cols (10) e Taylor \& Cols (14), o Tinidazol possui, "in vivo" e "in vitro", nítida atividade antiparasitária contra os seguintes agentes biológicos: Trichomonas vaginalis, Tri-

* Trabalho da disciplina de Doenças Infectuosas e Parasitárias do Departamento de Saúde Comunitária (Curso de Medicina do Setor de Ciências da Saúde da Universidade Federal do Paraná). Apresentado ao $X$ Congresso da Sociedade Brasileira de Medicina Tropical, Curitiba, Paraná, 3 a 6 Fevereiro de 1974. Caixa Postal, 1508, 80.000/Curitiba, Paraná, Brasil.

* Professor Titular.

** Professor Adjunto da Disciplina de Parasitologia do Departamento de Patologia Básica. Setor de Ciências Blológicas da Univ. Fed. do Paraná.

**** Monitores de Doenças Infectuosas e Parasitárias.

Recebido para publicaçāo em 5-11-1974. 
chomonas foetus, Entamoeba histolytica, Eimeria tenela e Histoplasma mallagridis. Estudos experimentais "in vitro" revelaram que o Tinidazol, como tricomonicida, é 4 a 16 vezes mais ativo do que o metronidazol.

Embora não tenham sido feitos estudos farmacológicos com relação à Giardia lamblia, sabe-se que os derivados nitroimidazólicos atuam também contra este protozoárío.

Os estudos clínico-terapêuticos feitos por Andersson \& Cols. (3) e Amato Neto \& Cols. (2) foram os únicos que precederam a presente investigação na giardíase e permitiram demonstrar a atividade giardicida do Tinidazol

O Tinidazol ou etil-2-(2-metil-5-nitro-1-imidazolil)-etil-sulfona tem a fórmula empírica $\mathrm{C}_{8} \mathrm{H}_{13} \mathrm{~N}_{3} \mathrm{O}_{4} \mathrm{~S}$.

\section{MATERIAL E MÉTODOS}

Esta investigação clínico-terapêutica refere-se ao tratamento de trinta pacientes parasitados pela Giardia lamblia, atendidos no ambulatório e nas enfermarias da Clínica de Doenças Infectuosas e Parasitárias do Hospital de Clínicas da Universidade Federal do Paraná e na clínica particular de um dos autores (M. C. B.).

A distribuição dos pacientes pelas idades e sexos está resumida na Tabela $I$.
As idades dos pacientes variaram de 5 a 60 anos. Houve predomínio de pacientes do grupo etário de 6 a 10 anos e do sexo feminino entre os quais não havia, como medida acauteladora, nenhuma gestante.

O medicamento foi usado em drágeas contendo $150 \mathrm{mg}$ de substância ativa.

Aos pacientes com idade superior a 10 anos o medicamento foi administrado na dose diária de $300 \mathrm{mg}$,em duas tomadas de uma drágea, após o desjejum e a janta, durante o prazo de cinco dias. AOs pacientes com idade até 10 anos a posologia diária era de $150 \mathrm{mg}$, isto é, uma drágea durante o mesmo prazo de 5 dias. As posologias totais foram, respectivamente, de $1500 \mathrm{mg}$ e de $750 \mathrm{mg}$, para os indivíduos com idade superior e inferior a 10 anos.

Para o diagnóstico e para o posterior controle de cura foram os pacientes submetidos a exames parasitológicos de fezes pelos métodos de Faust e cols., da centrífugo-flutuaçāo em solução de sulfato de zinco e de Hoffman, Pons e Janer da sedimentação espontânea em água.

Os controles parasitológicos foram realizados a partir de uma semana após o término da medicação e foram sempre em número de três, com intervalos de uma semana, no $70^{\circ}, 14 .^{\circ}$ e $21 .^{\circ}$ dia após o último dia de tratamento.

Foram considerados curados os pacientes que tiveram os três exames parasitológicos de controle negativos nos dois métodos de exame empregados.

TABELA I $\sim$ Distribuição dos pacientes segundo idade e sexo

\begin{tabular}{r|c|c|c}
\hline $\begin{array}{c}\text { Grupo } \\
\text { ctário } \\
\text { (anos) }\end{array}$ & Masculino & Feminino & Total \\
\cline { 2 - 4 } & & & \\
\hline $1-5$ & 1 & 1 & 2 \\
$6-10$ & 3 & 5 & 8 \\
$11-20$ & 3 & 4 & 7 \\
$21-30$ & 2 & 4 & 6 \\
$31-40$ & 2 & 3 & 5 \\
$41-50$ & - & 1 & 1 \\
$51-60$ & 1 & - & 1 \\
\hline
\end{tabular}


O controle de tratamento obedeceu assim às normas estabelecidas pelo Primeiro Encontro de Pesquisadores em Medicina Tropical, em São José dos Campos, São Paulo $(30 / 11$ e $1 \% / 12 / 973)$ e referendadas pelo $X$ Congresso da Sociedade Brasileira de Medicina Tropical, a 6 de fevereiro de 1974 (9).

Proibimos a ingestão de bebidas alcoólicas durante o período de tratamento, a fim de evitar eventuais reações que soem ocorrer nos indivíduos que ingerem concomitantemente nitriomidazois e álcool ruborização da face, cólicas intestinais, náuseas e vômitos.

\section{RESULTADOS}

Dos trinta pacientes tratados apenas um não apresentou negativação dos exames parasitológicos de controle no tocante à presença de cistos de Giardia lamblia. Conseguiu-se, destarte ,o elevado percentual de curas parasitológicas de $96,6 \%$.

Três pacientes infectados também pela Entamoeba histolytica tiveram os exames parasitológicos de controle negativados para este rizópodo .O pequeno número de casos não nos autoriza tirar qualquer conclusão a respeito.

A medicação foi muito bem tolerada, não se assinalando reações colaterais indesejáveis.

\section{COMENTÁRIOS E CONCLUSÕES}

Os resultados alcançados confirmam as duas investigações anteriores à nossa $(2,3)$, demonstrando que o Tinidazol é droga altamente eficaz no tratamento da giardíase. Certamente, virá ele ocupar lugar de destaque na terapêutica da protozoose, em que pese a existência anterior de giarcicidas bastante eficazes, como o metronidazol (15) e a nitrimidazina (5). Deriva tal conclusão da expressiva eficácia giardicida do Tinidazol ( $86,6 \%$ de curas) e da ausência de efeitos colaterais indesejáveis.
Fundamentados nos resultados da presente investigação clínico-terapêutica, destacamos como pontos importantes da nossa contribuição:

1 - No tocante à capacidade curativa do novo giardicida, o índice global de curas obtidas de $96,6 \%$ se situa entre aqueles obtidos por Andersson \& Cols. $(100 \%)$ e Amato Neto \& Cols. $(93,3 \%)$. Andersson \& Cols. (3) curaram com Tinidazol todos cs 24 estudantes suecos que se infectaram com Giardia lamblia em uma excursão a Leningrado (U.R.S.S.).

Evidentemente serão necessárias novas investigações com o Tinidazol na giardíase, de vez que é este o terceiro estudo publicado a respeito $(2,3)$.

2 - A redução do período de tratamento para cinco dias apenas, com posologias totais de $1500 \mathrm{mg}$ e $750 \mathrm{mg}$, respectivamente, em indivíduos com idade superior e inferior a 10 anos, prova que é possível diminuir a posologia empregada pelos dois grupos de pesquisadores que nos precederam. Andersson \& Cols. (3) e Amato Neto \& Cols. (2) utilizaram a droga durante prazo de uma semana e doses totais de substância ativa de $2100 \mathrm{ming}$. Contudo, em um caso em que foram obrigados a interromper o tratamento no quarto dia, Amato Neto \& Cols. (2) conseguiram curar a giardíase com posolcgia total bem menor (1200 $\mathrm{mg}$ ).

3 - A excelente tolerância do medicamento por parte dos pacientes tratados, na ausência de reaçōes colaterais indesejáveis.

\section{AGRADECIMENTOS}

Consignamos agradecimentos à "Pfizer Química Limitada" pelo fornecimento do Tinidazol para a presente investigaçāo.

\section{$S U M M A R Y$}

The Autors study the therapeutic efficacy of Tinidazole, a new nitrimidazole derivative, in the treatment of thirty patients with giardiasis.

They employed the daily dosage of $300 \mathrm{mg}$ for twenty patients and $150 \mathrm{mg}$ for ten children, respectively with ten years of age or and less during five consecutive days. 
The diagnosis and the parasitological evaluation of the results of treatment were accomplished by stool examinations using zinc sulphate centrifugal-flotation technique of Faust et al. and sedimentation-concentration technique of Hoffman, Pons and Janer. The control started one week after the treatment and consisted of three stool examinations, at one week intervals.

The Authors achieved 96.6 per cent of parasitological cures. They stressed the excellent tolerance of the drug, even by patients with serious fundamental diseases or by children. No side effects were registered.

They conclude that Tinidazole is an excellent drug against Giardia lamblia infections.

\section{REFERÊNCIAS BIBLIOGRAFICAS}

1. ALMEIDA, A.S.; NICULITCHEFF, G. X.; SANTOS, O.; DENIS, R. \& ZENI JR., J. - A respeito das enteroparasitcses humanas em Curitiba. Bol. Centro Debates Cient.-Cult. Dr. Victor do Amaral 1: 15-20, 1955.

2. AMATO NETO, V.; LEVI, G.C.; KONICHI, S.R. \& CAMPOS, L.L. - Eficácia do tinidazol no tratamento da giardíase. Rev. Soc. Bras. Med. Trop. 6: 147-149, 1972.

3. ANDERSSON, T.; FORSSEL, J. \& STERNER, G. - Outbreak of giardiasis: effect of a new antiflagellate drug, tinidazole. Brit. Med. J. 2: 449451, 1972.

4. BARANSKI, M.C.; LIMA, E.C. \& RIBEIRO, S.S. - Incidência das parasitoses intestinais entre os alunos do Serviço Nacional de Aprendizagem Industrial, em Curitiba. Bol. Centro Debates Cient.-Cult. Dr. Victor do Amaral 1: 21-29, 1955.

5. BARANSKI, M.C.; GOMES, N.R.; GODOY, O.F.; FONTOURA DA SILVA, A.; SZPEITER, N.; KOTAKA, P.I. \& SILVEIRA, H.B. - Tratamento da giardíase com nitrimidazina. Trib. Méd. 14: 36-40, 1972.

6. CARNEIRO F. ${ }^{\circ}$ M. - Enteroparasitoses no Hospital de Clínicas da Universidade Federal do Paraná. An. Fac. Med. Univ, Fed. Paraná 9-10: 173-191, 1966-1967.

7. HOWES JR., H.L.; LYNCH, J.E. \& KIVLIN, J.L. - Tinidazole a new antiprotozoal agent: effect on Trichomonas and other protozoa. Antimicrob. Agents Chemother 9: 261-266, 1970.

8. LIMA, E.C. \& BARANSKI, M.C. Incidência dos parasitos intestinais entre escolares de Curitiba. Rev. Méd. Paraná 22: 24-33, 1953.

9. METODOLOGIA PARA AVALIAÇÃO TERAPEUTICA DE DROGAS ANTIPARASITARIAS: I - PARASITOS INTESTINAIS. Primeiro Encontro de Pesquisadores em Medicina Tropical, 26 págs., 1973.

10. MILLER, M.W.; HOWES JR.; H.L. \& ENGLISH, A.R. - Tinidazole, a potent new antiprotozoal agent. Antimicrob. Agents Chemother. 1969, 9: 257-260, 1970.

11. MUNHOZ DA ROCHA, J.M. - Contribuição ao conhecimento dos parasitos intestinais em Curitiba. Tese doutoramento Fac. Med. Univ. Fed. Paraná, Pap. Requião, Curitiba, 1950.

12. RIBEIRO, S.S.; BARANSKI, M.C. \& LIMA, E.C. - Contribuição ao estudo do parasitismo intestinal em Curitiba. Rev. Dep. Saúde Paraná 3: 126-131, 1953.

13. TAKANO-MORON, J. - Giardiasis. In MARCIAL-ROJAS, R.A. - Pathology of Protozoal and Helminthic Diseases with Clinical Correlation. Baltimore, Williams \& Wilk ins Co., 1971, pp. 115-123.

14. TAYLOR, JR.; J.A.; MIGLIARDI, J.R. \& VON WITTENAU, M.S. Tinidazole, and metronidazole pharmacokinetics in man and mouse. $A n-$ timicrob. Agents Chemother. 1969, 9 : 267-270, 1970.

15. TREZUB, C.J.; CAGLIARI SANTOS, L.A.; CARNEIRO F..$^{\circ}$ \& BARANSKI, M.C. - Metronidazol em giardíase. Anais $\mathrm{X}$ Congresso Soc. Bras. Med. Trop., Curitiba, 3 a 6 fevereiro 1974, resumo 79. 